\title{
Medical History Supplemental Qualifiers Dataset
}

National Cancer Institute

\section{Source}

National Cancer Institute. Medical History Supplemental Qualifiers Dataset. NCI

Thesaurus. Code C147223.

A dataset containing supplemental information, specifically non-standard variables, to parent records in the medical history domain. 